\title{
Longitudinal simulations and measurements of stretched bunches
}

\author{
N. Towne \\ National Synchrotron Light Source, Brookhaven National Laboratory, Upton, New York 11973
}

(Received 6 February 2001; published 28 November 2001)

\begin{abstract}
Extending the method of Warnock and Ellison for the simulation of the Vlasov-Fokker-Planck equation for bunched beams [in Proceedings of the 2nd ICFA Advanced Accelerator Workshop on the Physics of High Brightness Beams, Los Angeles, CA, 1999 (World Scientific, Singapore, 2000)], we analyze arbitrary radio-frequency potentials and high- $Q$ impedances and study instabilities in stretched bunches. Results for the microwave instability driven by a broadband impedance and instability driven by high- $Q$ rf modes in stretched bunches are compared with measurements of the vacuum ultraviolet ring at the National Synchrotron Light Source. A method for the calculation of response functions is developed, and simulated response functions are compared to experimental results.
\end{abstract}

DOI: $10.1103 /$ PhysRevSTAB.4.114401

PACS numbers: $29.27 . \mathrm{Bd}$

\section{INTRODUCTION}

There has been considerable interest in recent years in the saturation and relaxation of longitudinal instabilities in storage rings. Instabilities driven by both broadband impedances [1-4] and high- $Q$ impedances [5] have been simulated and compared with instabilities in existing storage rings. These studies have shed considerable light on the limiting of these instabilities and highlighted the limits of linearized treatments of coherent modes and frequencies in bunched beams.

Bunched beams in which synchrotron frequency spread is introduced for Landau damping and lifetime improvement [6,7] have been simulated much less than unstretched bunches, although application of linearized methods to fully stretched bunches have had some success in describing coherent frequencies and stability boundaries [8-12]. But the limiting behavior of instabilities in stretched bunches beyond the linear approximation is less understood and deserves further study.

In this paper, we discuss computational methods and a new computer code capable of integrating the VlasovFokker-Planck (VFP) equation for non-Gaussian bunches and their application to the study of instabilities of stretched bunches. The methods are based on the work of Warnock and Ellison (W\&E) [1] extended to accommodate nonharmonic radio-frequency potentials and high- $Q$ impedances as well as a broadband impedance. Section II of this paper describes the computer code. A method for the calculation of beam response functions is developed (Sec. III) and demonstrated. Using the results of simulations, three-dimensional plots of the particle distribution in phase space, animated sequences of plots, and plots of bunch lengths, beam spectra, and phase and energy spread may also be generated.

Section IV describes certain instabilities observed in the National Synchrotron Light Source (NSLS) vacuum ultraviolet (VUV) ring. Section V discusses the results of simulation of saturation of certain instabilities as well as calculations of beam response functions for stretched bunches. These results are applied phenomenologically to instabilities observed in the NSLS VUV ring (Sec. IV) to help in understanding the ring and its collective phenomena. Specifically, we discuss (i) the microwave instability driven by a simple broadband impedance; (ii) calculations of the longitudinal beam-response function (BRF) and comparison with VUV-ring data; and (iii) simulations of instabilities driven by high- $Q$ and broadband impedances.

\section{THE COMPUTER CODE}

The computer code was written to numerically integrate the VFP equation in the time domain and calculate observable quantities from the solutions. Section II A gives the notation and definitions used in this paper. Section II B describes briefly the VFP equation and W\&E's [1] method of integrating it. Then Sec. IIC describes the calculation of the initial Haïssinski solution [13], which is used as an initial condition in the full calculation. The approach differs slightly from that of W\&E to overcome the lack of convergence of the recursive-substitution method when applied to stretched bunches of sufficiently high current. Section II D describes how high- $Q$ rf modes are integrated into the code.

\section{A. Notation, the equations of motion, and the Vlasov equation}

This section describes the definitions and notation used in this paper.

The phase coordinate $\phi$ is related to the azimuthal angle in the ring $\theta$ through

$$
\theta=\omega_{0} t+\phi
$$

where $\omega_{0}$ is the revolution frequency. So $\phi$ is comoving with the bunch, and greater $\phi$ means earlier in time. The single-particle Hamiltonian is

$$
H_{\mathrm{rf}}=H_{\mathrm{rf}}(\phi, p)=p^{2} / 2+U_{\mathrm{rf}}(\phi),
$$


where $p$ is the momentum variable conjugate to $\phi$, and Hamilton's equations are

$$
\begin{aligned}
d \phi / d t & =p, \\
d p / d t & =-d U_{\mathrm{rf}}(\phi) / d \phi .
\end{aligned}
$$

$U_{\text {rf }}$ is the rf potential in which the particles oscillate,

$$
U_{\mathrm{rf}}(\phi)=\frac{\alpha \omega_{0}}{E_{0}} \frac{e \omega_{0}}{2 \pi} \int^{\phi} d \phi V_{\mathrm{rf}}(\phi),
$$

where $\alpha$ is the momentum compaction, $E_{0}$ is the synchronous particle energy, $e$ is the electronic charge, and $V_{\mathrm{rf}}$ is the rf voltage in the cavities. The Hamiltonian $H_{\rho}=$ $H_{\rho}(\phi, p ; t)$ associated with a voltage $V=V_{\rho}(\phi ; t)$ is

$$
\begin{aligned}
H_{\rho}(\phi, p ; t) & =U_{\rho}(\phi ; t) \\
& =\frac{\alpha \omega_{0}}{E_{0}} \frac{e \omega_{0}}{2 \pi} \int^{\phi} d \phi V_{\rho}(\phi ; t) .
\end{aligned}
$$

$V_{\rho}$ is the voltage induced by a line density $\rho$ due to the short-range wake potential $W$,

$$
V_{\rho}(\phi ; t)=I_{\mathrm{av}} T_{0} \int_{-\pi}^{\pi} d \phi^{\prime} W\left(\phi-\phi^{\prime}\right) \rho\left(\phi^{\prime} ; t\right),
$$

where $I_{\mathrm{av}}$ is the average current, $I(\phi ; t)=2 \pi I_{\mathrm{av}} \rho(\phi ; t)$ is the instantaneous current, and $\rho$ is normalized so that $\int d \phi \rho=1$. The time dependence of the functions of Eqs. (2.5) and (2.6) represents the much slower turn-byturn evolution of the functions.

The VFP equation describes how distributions $\Psi$ of particles in phase space evolve in time.

$$
\partial \Psi / \partial t+\left\{\Psi, H_{\mathrm{rf}}+H_{\rho}\right\}=R_{\mathrm{FP}} \Psi,
$$

where the braces represent Poisson brackets,

$$
R_{\mathrm{FP}}=\partial_{p}\left(2 \beta p+D \partial_{p}\right),
$$

$\beta$ and $D$ are the damping and diffusion rates, and $\rho$ is related to the phase-space distribution $\Psi$ through

$$
\rho(\phi ; t)=\int d p \Psi(\phi, p ; t) .
$$

Let the operator $P$ be the operator that takes $\Psi$ to $\rho$, i.e., $\rho=P \Psi$. The Haïssinski distribution $\Psi_{\mathrm{Ha}}$ is that part of $\Psi$ that is stationary,

$$
\left\{\Psi_{\mathrm{Ha}}, H_{\mathrm{rf}}\right\}+\left\{\Psi_{\mathrm{Ha}}, H_{\rho \mathrm{Ha}}\right\}=R_{\mathrm{FP}} \Psi_{\mathrm{Ha}},
$$

and $\rho_{\mathrm{Ha}}=P \Psi_{\mathrm{Ha}}$. Let $H_{0}=H_{\mathrm{rf}}+H_{\rho \mathrm{Ha}}$. The remainder, $\delta \Psi=\Psi-\Psi_{\mathrm{Ha}}$, satisfies the equation

$$
\begin{aligned}
\partial_{t} \delta \Psi+\left\{\Psi_{\mathrm{Ha}}, H_{\delta \rho}\right\}+\left\{\delta \Psi, H_{0}\right\}+ \\
\left\{\delta \Psi, H_{\delta \rho}\right\}=R_{\mathrm{FP}} \delta \Psi,
\end{aligned}
$$

where $\delta \rho=P \delta \Psi$. This equation is nonlinear in the fourth term and is linearized by dropping this term. The second term is expanded to

$$
\left\{\Psi_{\mathrm{Ha}}, H_{\delta \rho}\right\}=-\left(\partial_{p} \Psi_{\mathrm{Ha}}\right) W \delta \rho,
$$

where the wake potential $W$ is acting on the line density $\delta \rho$ according to Eq. (2.6). Let :H: be the operator that maps a function $\Psi$ to $\{\Psi, H\}$. So now the linearized Eq. (2.11) is

$$
\partial_{t} \delta \Psi-\left(\partial_{p} \Psi_{\mathrm{Ha}}\right) W P \delta \Psi+: H: \delta \Psi=R_{\mathrm{FP}} \delta \Psi .
$$

The linearized Vlasov equation is Eq. (2.13) with the Fokker-Planck (FP) terms dropped. The potential-welldistortion term is $\left\{\delta \Psi, H_{\rho \mathrm{Ha}}\right\}$, which is part of the third term.

Let the time dependence of $\delta \Psi$ be harmonic with frequency $\Omega$. A homogeneous equation in the line density $\delta \rho$ is derived from Eq. (2.13) by inverting the sum of the operators of the first, third, and fourth terms and applying the inverted operators and $P$ to the equation on the left. This yields

$$
\delta \rho=G W \delta \rho,
$$

where $G=G(\Omega)$ is the frequency-domain beam transfer function (BTF), using Shaposhnikova's notation [14],

$$
G(\Omega)=P\left(i \Omega+: H:-R_{\mathrm{FP}}\right)^{-1}\left(\partial_{p} \Psi_{\mathrm{Ha}}\right) .
$$

One should not regard the linearized VFP equation as somehow being reduced to Eq. (2.14). Coherent frequencies determined by Eq. (2.14) are also coherent frequencies determined by Eq. (2.13) but the converse is not necessarily true. We know on physical grounds that there exists a response function $G$ mapping voltages to line densities as in

$$
\delta \rho=G V,
$$

where $V$ is the total field seen by the bunch, and we know that the VFP equation is capable of determining $G$ due to the completeness of the VFP equation. So the question of how to calculate $G$ of Eq. (2.16) from the VFP equation is a meaningful one even if Eq. (2.14) is not complete. This is to be expected since functions $V(\phi ; t)$ that excite the bunch do not excite all degrees of freedom since only certain functions $\delta \Psi$ map to the special class of functions $\left(\partial_{p} \Psi_{\mathrm{Ha}}\right) V(\phi ; t)$ under the action of $i \Omega+$ $: H:-R_{\mathrm{FP}}$. Furthermore, not all degrees of freedom of the bunch are distinguishable in line density projections. Both : $H_{0}$ : and $R_{\mathrm{FP}}$ are unbounded operators and questions of the existence of the inverse of $\left(i \Omega+: H_{0}:-R_{\mathrm{FP}}\right)^{-1}$ in Eq. (2.15), its domain and range, and how to calculate it in any given basis are not resolved at this time. A closed-form solution to Eq. (2.15) may not even exist. But note that some of these concerns also apply to the Vlasov equation without the potential-well-distortion term and to Krinsky 
and Wang's $(\mathrm{K} \& \mathrm{~W})$ successful calculation of $G$ in that restricted context [15].

The total voltage $V$ is considered to be a superposition of a voltage $V_{\text {ext }}$ externally applied to the ring and $W \delta \rho$, i.e.,

$$
V=W \delta \rho+V_{\mathrm{ext}} .
$$

In this case, Eq. (2.16) becomes inhomogeneous,

$$
\delta \rho=G\left(W \delta \rho+V_{\mathrm{ext}}\right) .
$$

An equation in $V$ is readily derived from Eqs. (2.17) and (2.16),

$$
V=W G V+V_{\mathrm{ext}} .
$$

The derivation of the BTF given by Krinsky and Wang [15] and Shaposhnikova [14] drop both the FP and the potentialwell-distortion terms. Note that $G(\Omega)$ in this case is unbounded when $\Omega$ is real: Normalizable line densities are not all mapped to normalizable line densities. For this reason, the operator $G W$ is not absolutely continuous for real $\Omega$ since $G$ is unbounded. Consequently, Fredholm theory cannot be applied directly to Eq. (2.18). But it is an open question whether an operator related to $G W$ by a similarity transformation is absolutely continuous. $\mathrm{K} \& \mathrm{~W}$ 's result [15] is expressed in a basis of azimuthal harmonics. They introduce the matrix elements $F_{m n} \equiv-G_{m n} / \kappa$, where $\kappa$ is a constant.

It is useful to define the frequency-domain longitudinal beam response function $T=T(\Omega)$, which relates $V_{\text {ext }}$ to the line density it induces in the presence of finite current and ring impedance, i.e.,

$$
\delta \rho=T V_{\mathrm{ext}}
$$

By solving Eq. (2.18) formally for $T$, we find

$$
T=(1-G W)^{-1} G \text {. }
$$

(Do not confuse $T$ with K\&W's $T=G Z$, where $Z$ is the Fourier transform of the wake potential $W$.)

\section{B. Solution of the Vlasov-Fokker-Planck equation}

In W\&E's method [1], the two components of the Hamiltonian $H$, the rf Hamiltonian $H_{\mathrm{rf}}$, and the $\rho$ perturbation $H_{\rho}$, generate the maps $M_{\mathrm{rf}}(\Delta t)$ and $M_{\rho}(\Delta t)$ carrying points of phase space through a given time step $\Delta t$ according to the equations of motion. Using the terms of tensor analysis, these maps are the flows associated with the vector fields $: H_{\mathrm{rf}}:$ and $: H_{\rho}$ :, respectively, evaluated at the time step $\Delta t$. Each map is symplectic and hence volume preserving. These maps in turn define Perron-Frobenius (PF) operators $\mathcal{M}_{\text {rf }}$ and $\mathcal{M}_{\rho}$ that step a function $\Psi$ convectively according to the inverse of the corresponding map:

$$
\begin{gathered}
\mathcal{M}_{\mathrm{rf}}\{\Psi\}(x)=\Psi\left(M_{\mathrm{rf}}^{-1}(x)\right), \\
\mathcal{M}_{\rho}\{\Psi\}(x)=\Psi\left(M_{\rho}^{-1}(x)\right) .
\end{gathered}
$$

The $\Delta t$ dependence is suppressed. Interpolation is used since the mapped grid points do not coincide with the original grid points. W\&E's method for time stepping Eq. (2.7) is to apply the three operators $\mathcal{M}_{\text {rf }}, \mathcal{M}_{\rho}$, and the Fokker-Planck terms on the right-hand side of Eq. (2.7) in sequence for each time step. All this is done on a grid of points in the phase space in which $\Psi$ is defined. Their paper describes this process more fully.

$\mathrm{W} \& \mathrm{E}$ assume that the rf potential is quadratic, i.e., that the (small charge) bunch is Gaussian in shape. In this case, the map $M_{\mathrm{rf}}(\Delta t)$ is simply a rotation of the phase space. This map is generalized to arbitrary rf potentials by calculating $M_{\mathrm{rf}}^{-1}(\Delta t)$ at every grid point by integrating Hamilton's equations for the rf Hamiltonian through time $-\Delta t$. Hamiltonians with non-Gaussian momentum dependence may also be accommodated. Although this generalization requires more calculation, the map is ordinarily computed only once and saved on the same grid on which $\Psi$ is defined. Overall, the code is not excessively slowed by the calculation of the map. This generalization to arbitrary rf potentials is the first way in which this code extends W\&E's methods.

The division of the exact PF operator Eq. (2.7) into the two PF operators $\mathcal{M}_{\text {rf }}$ and $\mathcal{M}_{\rho}$ is not unique. In contrast to $\mathrm{W} \& \mathrm{E}$, one can write the Hamiltonian not as the sum of $H_{\mathrm{rf}}$ and $H_{\rho}$ but rather as the sum of $H_{0}$ and $H_{\delta \rho}$. This change is suggested by writing the nonlinear VFP equation (2.11)

$$
\partial \Psi / \partial t+\left\{\Psi, H_{0}+H_{\delta \rho}\right\}=R_{\mathrm{FP}} \Psi
$$

Corresponding to the Hamiltonians $H_{0}$ and $H_{\delta \rho}$ are the PF operators $\mathcal{M}_{0}$ and $\mathcal{M}_{\delta \rho}$ derived as described above. But now the Haïssinski distribution in the phase space is stationary with respect to $\mathcal{M}_{0}$, and $\mathcal{M}_{\delta \rho}$ is regarded as a perturbation. This is the second way in which the code departs from the methods of W\&E. Note that our division of the Hamiltonians is not clearly superior since, in both cases, $\delta \rho$ is not small when working with largeamplitude instabilities. Only when the perturbation $\delta \rho$ is small is $\mathcal{M}_{\delta \rho}$ small. In the linear growth stage, when calculating linear beam response functions, this decomposition has a clear computational advantage. The asymmetry in the equilibrium distribution induced by a broadband impedance suggests that the use of $\mathcal{M}_{0}$ may result in perturbations with less gross asymmetry so that $\mathcal{M}_{\delta \rho}$ has to do less work mapping the phase space onto itself.

\section{Haïssinski solution}

The calculation of the Haïssinski solution [13] for stretched bunches is discussed in some detail here because of difficulty with convergence. In the usual method of recursive substitution, a sequence of line densities $\rho_{0 n}(\phi)$ is generated according to 


$$
\rho_{0 n+1}(\phi)=\frac{e^{-\left[U_{\mathrm{rf}}(\phi)+H_{\rho_{0 n}}(\phi)\right] / \sigma^{2}}}{\int_{-\infty}^{\infty} d \phi^{\prime} e^{-\left[H_{\mathrm{rf}}\left(\phi^{\prime}\right)+H_{\rho 0 n}\left(\phi^{\prime}\right)\right] / \sigma^{2}}},
$$

where $\sigma$ is a constant defined in Ref. [15]. When this sequence converges, it converges to the Haïssinski line density $\rho_{\text {На }}$. Unfortunately, it fails to converge at singlebunch currents of interest when applied to stretched VUV ring bunches. To get around this problem, the weighted average

$$
\begin{aligned}
\rho_{0 n+1}(\phi)= & w \frac{e^{-\left[U_{\mathrm{rf}}(\phi)+H_{\rho 0 n}(\phi)\right] / \sigma^{2}}}{\int_{-\infty}^{\infty} d \phi^{\prime} e^{-\left[H_{\mathrm{rf}}\left(\phi^{\prime}\right)+H_{\rho 0 n}\left(\phi^{\prime}\right)\right] / \sigma^{2}}} \\
& +(1-w) \rho_{0 n}(\phi)
\end{aligned}
$$

was used. Unfortunately, for high current, the weight $w$ must be small and the convergence is very slow. Because the convergence is so slow at high current, the typical calculation cannot proceed to machine precision. This is not a hardship when calculating instabilities since the error serves to seed an instability in a simulation if the bunch is unstable, or simply to generate an initial transient in a stable bunch. However, when calculating a response function in a stable bunch, the transient caused by this error is mixed with the transient associated with the initial impulse used in the calculation (Sec. III) introducing an error in the response-function calculation. To overcome this problem, another calculation without the applied impulse is performed and used to subtract the unwanted transient from the calculations including the applied impulses.

In our approach, the calculated Haïssinski distribution does not include the distortion to the rf potential well caused by beam loading of high- $Q$ modes. Only the components of the ring wake potential having short range are included in the calculation of $H_{\rho \mathrm{Ha}}$. Any distortion of the potential well caused by higher-order modes or other high- $Q$ resonances in the ring impedance are assumed small and are absorbed (approximately) into the externally prescribed rf potential.

\section{High- $Q$ rf modes}

The rf-cavity modes receive periodic kicks from the succession of bunch passages through the cavities. In this paper, the evolution of the rf-mode excitations are described by discreet-time equations relating mode voltages on successive turns to the kicks they receive during a bunch passage. Most workers, however, describe the evolution of mode excitations using continuous-time differential equations; the discreet-time approach, while more natural [16], is less well known. While the discreet-time approach is far from new [5,17], the specific form of the difference equation used and its derivation are given here for clarity.

The beam current at azimuthal angle in the ring $\theta=$ $\omega_{0} t+\phi=2 \pi l$ is given by

$$
I(t)=2 \pi I_{\mathrm{av}} \rho(\phi ; t),
$$

where $I_{\mathrm{av}}$ is the average beam current and $l$ is an integer. The normalization of $\rho$ is chosen so that $\int \rho d \phi=1$. Since $\rho(\phi ; t)$ is a slowly changing function of $t$, the convolution integral,

$$
V(t)=\int_{-\infty}^{t} d t^{\prime} W\left(t-t^{\prime}\right) I\left(t^{\prime}\right),
$$

is divided into a $t^{\prime}$ integral over the range $\left(l^{\prime}-1\right) T_{0} \leq$ $t^{\prime} \leq l^{\prime} T_{0}$ and a sum over $-\infty<l^{\prime} \leq l-1$ plus a remainder corresponding to the current passage of the bunch through the cavity $\left(l^{\prime}=l\right)$. Setting the integration variable to $\phi^{\prime}=-\omega_{0} t^{\prime}$,

$$
\begin{aligned}
V\left(\phi, l T_{0}\right)= & 2 \pi I_{\mathrm{av}} \sum_{l^{\prime}=-\infty}^{l-1} \int_{\pi}^{-\pi} \frac{d \phi^{\prime}}{-\omega_{0}} W\left[\left(l-l^{\prime}\right) T_{0}-\frac{\phi-\phi^{\prime}}{\omega_{0}}\right] \rho\left(\phi^{\prime}, l^{\prime} T_{0}\right) \\
& +2 \pi I_{\mathrm{av}} \int_{\pi}^{\phi} \frac{d \phi^{\prime}}{-\omega_{0}} W\left(-\frac{\phi-\phi^{\prime}}{\omega_{0}}\right) \rho\left(\phi^{\prime}, l T_{0}\right),
\end{aligned}
$$

$V$ is divided into these two terms so that a particular approximation may be applied later. In Eq. (2.28), the fast time dependence of $V$ seen by the bunch as it crosses the kicker is parametrized by $\phi$, and the slow time dependence of $V$ is parametrized by $t=l T_{0}$ [see the discussion leading to Eq. (3.3)].

The wake potential for a resonator is [18]

$$
W(t)=\frac{k}{\cos \vartheta} e^{-i(\Omega t+\vartheta)}+\text { c.c. },
$$

where $\Omega=\bar{\omega}+i \Gamma$,

$$
\bar{\omega}=\sqrt{\omega_{r}^{2}-\Gamma^{2}},
$$

$\omega_{r}$ is the resonant frequency of the rf mode, the loss factor $k$ is the product of the damping rate $\Gamma=\omega_{r} / 2 Q$ and the rf-mode impedance $R, Q$ is the $Q$ of the mode,

$$
\tan \vartheta=\Gamma / \bar{\omega},
$$

and "c.c." represents the complex conjugate of the preceding expression. When this wake potential is inserted into Eq. (2.28), the $\phi$ integrals have kernel $e^{i \Omega\left(\phi-\phi^{\prime}\right) / \omega_{0}}$. Since the bunches are short and centered on zero, the kernel can be approximated by $e^{i h\left(\phi-\phi^{\prime}\right)}$, where $h$ is the harmonic number of the revolution frequency near which the rf mode resides, as long as the magnitude of $\Omega-h \omega_{0}$ times the bunch length (in time) is small. Changing variable from $l^{\prime}$ to $l^{\prime \prime}=l-l^{\prime}$, Eq. (2.28) becomes 


$$
\begin{aligned}
V\left(\phi, l T_{0}\right) \simeq & \frac{k T_{0} I_{\mathrm{av}}}{\cos \vartheta} e^{-i \vartheta} \sum_{l^{\prime \prime}=1}^{\infty} e^{-i \Omega l^{\prime \prime} T_{0}} \int_{-\pi}^{\pi} d \phi^{\prime} e^{i h\left(\phi-\phi^{\prime}\right)} \rho\left(\phi^{\prime},\left(l-l^{\prime \prime}\right) T_{0}\right) \\
& +\frac{k T_{0} I_{\mathrm{av}}}{\cos \vartheta} e^{-i \vartheta} \int_{\phi}^{\pi} d \phi^{\prime} e^{i h\left(\phi-\phi^{\prime}\right)} \rho\left(\phi^{\prime}, l T_{0}\right)+\text { c.c. }
\end{aligned}
$$

The first integral is $2 \pi e^{i h \phi} \rho_{h}\left(\left(l-l^{\prime \prime}\right) T_{0}\right)$, where $\rho_{h}\left(\left(l-l^{\prime \prime}\right) T_{0}\right)$ is the $h$ th Fourier coefficient of $\rho(\phi ; t)$ with respect to the variable $\phi$. The Fourier transform $\mathcal{F}_{m}$ with respect to $\phi$ of a function $f(\phi)$ is defined as

$$
\mathcal{F}_{m}\{\rho(\phi ; t)\} \equiv \delta \rho_{m}(t)=\frac{1}{2 \pi} \int_{0}^{2 \pi} d \phi \rho(\phi ; t) e^{-i m \phi} .
$$

The second term of Eq. (2.32) is the voltage in the bunch induced by the same bunch passage. Since the rf mode has high- $Q$, the intensity of $V$ is dominated by the accumulation of kicks the rf mode receives from many turns. For this reason, the bunch's immediate influence on $V$ may be neglected or modified. This justifies the last approximation, which is to replace the $\phi$ limit of integration in the second integral of Eq. (2.32) by $-\pi$. After defining $z=e^{i \Omega T_{0}}$, Eq. (2.32) becomes

$$
\begin{aligned}
V\left(\phi, l T_{0}\right) \simeq & 2 \pi \frac{k T_{0} I_{\mathrm{av}}}{\cos \vartheta} e^{-i \vartheta} e^{i h \phi} \\
& \times \sum_{l^{\prime \prime}=0}^{\infty} z^{-l^{\prime \prime}} \rho_{h}\left(\left(l-l^{\prime \prime}\right) T_{0}\right)+\text { c.c. }
\end{aligned}
$$

Let $\hat{V}_{l}$ be the first complex-valued term on the right-hand side of Eq. (2.34) but without the phase-dependent factor $e^{i h \phi}$. We see immediately that $\hat{V}_{l}$ evolves discreetly according to the recurrence relation

$$
\hat{V}_{l}=z^{-1} \hat{V}_{l-1}+k^{\prime} \rho_{h}\left(l T_{0}\right),
$$

where

$$
k^{\prime}=2 \pi \frac{k T_{0} I_{\mathrm{av}}}{\cos \vartheta} e^{-i \vartheta} .
$$

Equation (2.35) is used in the computer code to calculate the evolution of rf-mode amplitudes. The voltage that actually kicks the bunch is

$$
V(\phi ; t)=e^{i h \phi} \hat{V}_{l}+\text { c.c. }
$$

Note that, if the mode has $Q \gg 1, \tan \vartheta \ll 1$.

As was mentioned in Sec. II B, the code calculates field perturbations from $\delta \rho$ rather than from $\rho$. The total voltage seen by the bunch is the sum of the terms $V(\phi ; t)$ for the rf modes and the term for the broadband impedance-each constructed from the perturbation $\delta \rho$. This sum is used to construct the PF operator termed $\mathcal{M}_{\delta \rho}$ above. Note that, in constructing $\mathcal{M}_{\delta \rho}$ in this way, static beam-induced perturbations of the rf potential due to higher-order-mode (HOM) impedances are, in effect, absorbed by $U_{\text {rf }}$. No HOM impedances were included, however, in calculations described in this paper.

The discreet-time equation (2.35) is appropriate for describing the evolution of $V$ since, physically, the rf mode receives a kick of short duration from the bunch moment $\rho_{h}$ (or $\delta \rho_{h}$ ) every turn. Continuous-time equations are not superior to Eq. (2.35).

The time step $\Delta t$ of the integration of the VFP equation in calculations described in this paper was greater than $T_{0}$. So $\delta \rho_{h}$ inserted into Eq. (2.35) in place of $\rho_{h}$ is held constant during the iterations of Eq. (2.35) corresponding to the time step. The precise use of the discreet-time equation (2.35) requires that the time step $\Delta t$ of a simulation be a multiple of the revolution period. Although this requirement was not enforced, with the time step used, errors in the effective impedances of the modes were only $2 \%$.

\section{E. Performance}

Simulations were done using grids with between $50^{2}$ and $100^{2}$ grid points. W\&E used second-order interpolators in the function-interpolation implementation of the PF operators. Here, either third- or fifth-order interpolators were found to better preserve normalization. It was also found necessary to calculate the symplectic map $M_{0}(-\Delta t)$ for the time step to high precision and accuracy.

The grids used have many fewer points than those of W\&E. In spite of this, the normalization of the distribution function $\Psi$ was retained to a degree comparable to that reported by W\&E. Loss of normalization was usually due to nonnegligible value of the distribution function at the edge of the grid. This is the reason to have large spans of phase and energy as they can prevent significant particle densities from reaching the edge.

The computer code was written in MATHEMATICA permitting relatively quick program development. A significant penalty in computing time is paid for this, however, as MATHEMATICA code is interpreted. But, in MATHEMATICA, the elementary operation is often a list operation, which we found in simple numerical benchmarks to be only a factor of 3 slower than native code on a PC.

\section{BEAM RESPONSE FUNCTIONS}

A bunch's response to an external excitation, whether it is the response to a modulation of an rf system, an impulse, or the response to an rf frequency applied through a broadband device, contains detailed information about the bunch and its environment. While the evolution of a bunch through the VFP equation is nonlinear, the approximately linear response to a sufficiently small excitation is 
contained in the BRF $T$ of Eqs. (2.20) and (2.21) and reflects both the wake potential of the ring and the BTF $G$ [Eq. (2.15)] characterizing the bunch in the absence of an external potential $[14,15]$. The matrix elements $T_{m n}$, defined in the azimuthal-harmonic space on which the matrix elements $G_{m n}$ are defined, are often regarded as functions of frequency $\Omega$. They relate the Fourier transform $\tilde{\rho}_{m}(\Omega)$ of the line density to voltages $V_{\text {ext }}(t)$ applied at a frequency $\Omega+n \omega_{0}$. The BRF in the frequency domain, like the BTF, is readily and frequently measured; so it is useful to compute BRFs from time-domain simulations to compare with measured frequency-domain responses. Time-domain simulations can, in principle, be used to simulate a bunch stimulated by a continuous-wave rf voltage so as to simulate frequency-domain measurements. But each simulation determines a response function at only one frequency and must be long enough to accurately estimate the steady-state response. For these reasons, this approach is prohibitive. So there is reason to extrapolate the use of impulse responses for the determination of frequency-domain responses from linear network theory to the time-domain simulation of bunches. We note, however, that the impulse response of a bunch is complicated by the fact that a bunch is represented by a function of both time and phase. This section discusses how $T_{m n}(\Omega)$ can be calculated from suitable impulse responses.

The frequency-domain response functions are measured by exciting the bunch with a voltage that has a steady sinusoidal time dependence at a frequency $\omega=\Omega+n \omega_{0}$, i.e., $V_{\text {ext }}(t) \propto e^{-i\left(\Omega+n \omega_{0}\right) t}$, where $|\Omega| \ll \omega_{0}$. Since $\phi$ is a comoving coordinate such that $\theta=\omega_{0} t+\phi=2 \pi l(l$ is an integer) where the pickup and kicker are located, this time dependence is written

$$
V_{\text {ext }}\left(\phi ; t=l T_{0}\right)=V_{0} e^{-i(\Omega t-n \phi)},
$$

where $V_{0}$ is the peak voltage and $t=l T_{0}$ picks up the slow, and $\phi$ the fast, time dependence of $V_{\text {ext }}(t)$. This approximate form is justified by expanding

$$
\begin{aligned}
e^{-i\left(\Omega+n \omega_{0}\right) t} & =e^{-i\left(\Omega+n \omega_{0}\right)(2 \pi l-\phi) / \omega_{0}} \\
& =e^{-i \Omega l T_{0}} e^{i \Omega \phi / \omega_{0}} e^{i n \phi} .
\end{aligned}
$$

We are concerned only with the effect of $V_{\text {ext }}$ on the bunch, i.e., where $\phi \approx \omega_{0} \sigma_{t}$ ( $\sigma_{t}$ is the bunch length in time). Therefore, while $|\Omega| \ll \omega_{0},\left|\Omega \sigma_{t}\right| \ll 1$ holds and the middle exponential can be approximated by unity. Using the same reasoning and the same condition, the discreet $l T_{0}$ in the first exponential can be replaced by the continuous variable $t$,

$$
e^{-i\left(\Omega+n \omega_{0}\right) t} \simeq e^{-i \Omega t} e^{i n \phi} .
$$

Let us now discuss how the VFP equation determines the evolution of transients and impulse responses in stable bunches. Suppose a voltage $V_{\text {ext }}(\phi ; t)$ with the property that $V_{\text {ext }}$ goes to zero at $t=-\infty$ is applied to a bunch using a kicker. The initial/boundary condition imposed on the bunch is that at $t=-\infty, \Psi$ is the stationary Haïssinski distribution [13] $\Psi_{\mathrm{Ha}}$. The bunch then evolves according to the linearized VFP equation (2.13) giving a perturbed distribution

$$
\delta \Psi(\phi, p ; t)=B_{\mathrm{VFP}}\left\{V_{\mathrm{ext}}(\phi ; t)\right\},
$$

where $B_{\mathrm{VFP}}$ is the linear operator mapping functions $V_{\text {ext }}(\phi ; t)$ to phase space densities $\delta \Psi(\phi, p ; t)$ via the VFP equation. Note that it is essential that the bunch be stable; otherwise there is exponential growth of $\delta \Psi$ in a manner that is sensitive to how $\delta \Psi$ approaches zero as $t \rightarrow-\infty$.

The operator $P$ [defined following Eq. (2.9)] is applied to give us the line density as a function of time

$$
\delta \rho(\phi ; t)=P B_{\mathrm{VFP}}\left\{V_{\mathrm{ext}}(\phi ; t)\right\} .
$$

The operator $P B_{\mathrm{VFP}}$ is a representation of the BRF $T$ of Eq. (2.20). We can write down an integral transform for this representation knowing that the linearized VFP equation is time independent due to the time-independent single-particle Hamiltonian dynamics on the $\phi$ - $p$ phase space. It is

$\delta \rho(\phi ; t)=\int_{0}^{2 \pi} d \phi^{\prime} \int_{-\infty}^{\infty} d t^{\prime} T\left(\phi, \phi^{\prime} ; t-t^{\prime}\right) V_{\mathrm{ext}}\left(\phi^{\prime} ; t\right)$,

having the kernel $T\left(\phi, \phi^{\prime} ; t-t^{\prime}\right)$. Other representations of $T$ will be identified in this section; to simplify notation, the kernels of these representations will all be symbolized by " $T$," but will be distinguished by their arguments, e.g., $T_{m n}(\Omega)$. When Eq. (3.6) is Fourier transformed with respect to $\phi$ through $\mathcal{F}_{m}$ defined in Eq. (2.33), we have the transform

$$
\delta \rho_{m}(t)=\int_{-\infty}^{\infty} d t^{\prime} \sum_{n=-\infty}^{\infty} T_{m n}\left(t-t^{\prime}\right) V_{\mathrm{ext}, n}\left(t^{\prime}\right)
$$

with kernel $T_{m n}(t)$. When Eq. (3.6) is Fourier transformed with respect to $t$, we have

$$
\delta \rho(\phi ; \Omega)=\int_{0}^{2 \pi} d \phi^{\prime} T\left(\phi, \phi^{\prime} ; \Omega\right) V_{\mathrm{ext}}\left(\phi^{\prime} ; \Omega\right),
$$

and when Fourier transformed with respect to both $\phi$ and $t$,

$$
\delta \rho_{m}(\Omega)=\sum_{n=-\infty}^{\infty} T_{m n}(\Omega) V_{\mathrm{ext}, n}(\Omega) .
$$

Now we look at special forms of the function $V_{\text {ext }}$ that are both convenient for time-domain simulations and pertinent to the calculation of $T_{m n}(\Omega)$. Let the form of $V_{\text {ext }}$ be

$$
V_{\text {ext }}(\phi ; t)=f(\phi) \delta(t),
$$

where $f$ is an arbitrary phase dependence and $\delta(t)$ is the Dirac delta function. For $t<0$, the solution $\delta \Psi=\delta \rho=$ 0 by the boundary condition at $t=-\infty$ and the assumption of stability. This form of $V_{\text {ext }}$ is a single-turn impulse 
applied to the bunch so as to impart an energy kick to the Haïssinski distribution, having its $\phi$ dependence given by $f(\phi)$. [It is not the delta function $V_{\text {ext }}(t) \propto \delta(t)$ imparting a kick to one azimuthal point of a turn of the bunch.] Thus, at time zero, the bunch begins its phase-space evolution with an energy deviation determined by $f(\phi)$. This is convenient for a time-domain calculation since there is a well-defined initial condition at the starting time.

While $g(t)=\delta(t)$ is convenient to simulate, $f(\phi)=$ $e^{i n \phi}$ is pertinent to the calculation of $T_{m n}(\Omega)$ through the $\phi$ dependence of Eq. (3.3). To see this, insert $V_{\text {ext }}(\phi ; t)=$ $e^{i n \phi} \delta(t)$ into Eq. (3.6). With this forcing function, the result of a simulation produces the particular line density solution denoted $\delta \rho\left[e^{i n \phi} \delta(t)\right](\phi ; t)$, which is the response of the bunch to the delta-function excitation having the phase dependence $e^{i n \phi}$ also present in Eq. (3.3). By Fourier transforming with respect to $\phi$, we get, through Eq. (3.7), the response function

$$
T_{m n}(t)=\mathcal{F}_{m}\left\{\delta \rho\left[e^{i n \phi} \delta(t)\right](\phi ; t)\right\} .
$$

Finally, by Fourier transforming with respect to $t$ we get the frequency-domain response function

$$
T_{m n}(\Omega)=\mathcal{F}_{\Omega} \mathcal{F}_{m}\left\{\delta \rho\left[e^{i n \phi} \delta(t)\right](\phi ; t)\right\},
$$

where $\mathcal{F}_{\Omega}$ is the Fourier transform with respect to time whose action on a function $g(t)$ is

$$
\mathcal{F}_{\Omega}\{g(t)\} \equiv \frac{1}{2 \pi} \int_{-\infty}^{\infty} d t e^{i \Omega t} g(t)
$$

This is not quite the end of the story when the computer code integrating the VFP equation requires real forcing functions. In this case the function $f(\phi)=e^{i n \phi}$ must be broken up into $\cos n \phi+i \sin n \phi$ with each term a forcing term in a separate integration. By linearity, the results of the integrations can be superimposed to give

$$
\begin{aligned}
T_{m n}(t)=\mathcal{F}_{m}\{\delta \rho[\cos n \phi \delta(t)](\phi ; t) \\
+i \delta \rho[\sin n \phi \delta(t)](\phi ; t)\},
\end{aligned}
$$

and their Fourier transforms

$$
T_{m n}(\Omega)=\mathcal{F}_{\Omega}\left\{T_{m n}(t)\right\} .
$$

Equation (3.15) is the expression used to calculate beam response functions $T_{m n}(\Omega)$ from a time-domain code. Two calculations are performed starting with stable bunches with Haïssinski distributions. The Haïssinski distribution of one calculation is given an initial momentum kick with $\cos n \phi$ phase dependence, and the Haïssinski distribution of the other a $\sin n \phi$ phase dependence. The two linedensity functions of time resulting from the simulations are each Fourier transformed with respect to $\phi$ at harmonic $m$ and combined according to Eq. (3.14). The result is then Fourier transformed with respect to time to obtain $T_{m n}(\Omega)$ in Eq. (3.15).

Figure 1 shows an example of a beam response function calculated using the method described in this section. The impedance of the ring includes the main-cavity and

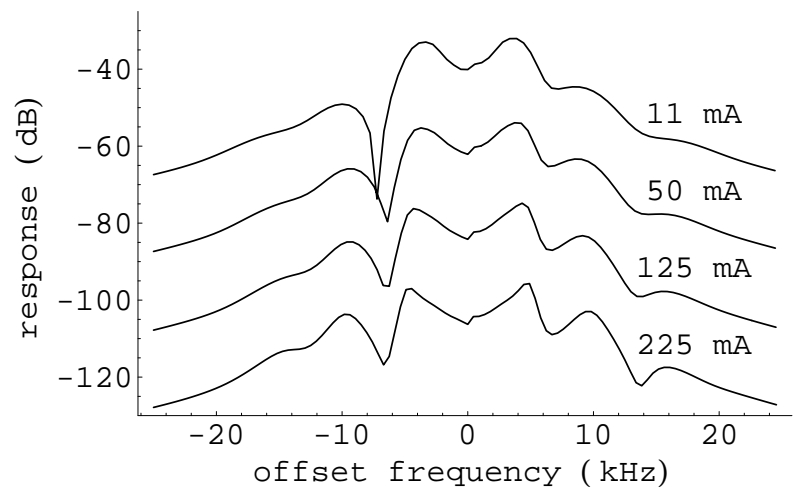

FIG. 1. Simulated beam response functions $T_{955}, T_{18}{ }_{55}, T_{3655}$, $T_{5555}$, and $T_{13555}$ (top to bottom) for a fully stretched $500 \mathrm{~mA}$ bunch in the National Synchrotron Light Source (NSLS) vacuum ultraviolet (VUV) ring. Realistically detuned main-cavity and harmonic-cavity impedances are included in the simulation. The vertical scale has an arbitrary scale factor. Machine parameters are given in Table I.

TABLE I. Values of VUV ring and main-cavity and harmoniccavity parameters (separated by a forward slash), symbols, and values.

\begin{tabular}{lcc}
\hline \hline \multicolumn{1}{c}{ Parameter } & Symbol & Value \\
\hline Synchronous energy & $E_{0}$ & $800 \mathrm{MeV}$ \\
Energy loss per turn & $U_{0}$ & $20.4 \mathrm{keV}$ \\
Momentum compaction & $\alpha$ & 0.0245 \\
Revolution frequency & $\omega_{0}$ & $2 \pi \times 5.8763 \mathrm{MHz}$ \\
Radiation damping rate & $\beta$ & $0.143 \mathrm{~ms}^{-1}$ \\
Fractional energy spread & $\sigma_{\epsilon}$ & $5 \times 10^{-4}$ \\
rf harmonic numbers & $h$ & $9 / 36$ \\
rf peak voltages & $V_{9} / V_{36}$ & $80 / 19.7 \mathrm{kV}$ \\
rf phases & $\psi_{9} / \psi_{36}$ & $74.2^{\circ} /-90^{\circ}$ \\
rf cavity impedances & $R_{9} / R_{36}$ & $435 / 100 \mathrm{k} \Omega$ \\
Loaded quality factors & $Q_{9} / Q_{36}$ & $6800 / 3360$ \\
Broadband impedance & $\left|Z_{n} / n\right|$ & $1.84 \Omega$ \\
\hline \hline
\end{tabular}

harmonic-cavity accelerating-mode impedances with realistic detuning for beam-loading compensation.

\section{MEASUREMENTS OF STRETCHED BUNCHES IN THE NSLS VUV RING}

The code described in this paper was developed to aid in the understanding of certain instabilities observed in the VUV ring. Two aspects of the collective behavior in the VUV ring have been partially illuminated by the code. The first is that, at higher current with stretched or overstretched bunches, the bunch becomes mildly unstable with the appearance of synchrotron sidebands with broad spectral bandwidth. At a given current, a partly stretched bunch may have these bands, and more stretching (increased harmonic-cavity power) increases the widths of the bands and the intensity of the instability. Overstretched bunches, i.e., bunches in a potential well with two minima, have lower threshold for this instability than stretched or understretched bunches. Furthermore, this instability is most 


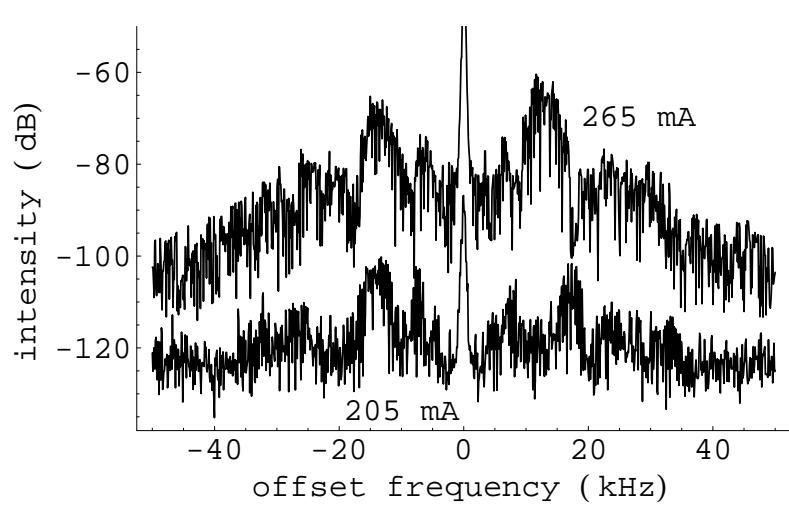

FIG. 2. Measured synchrotron sidebands of stretched singlebunch VUV beams at two beam currents about the 135th revolution harmonic. Machine parameters are given in Table I.

sensitive to single-bunch current and not total current. Figure 2 shows spectra of single-bunch beams in the VUV ring at 200 and $265 \mathrm{~mA}$ current, which is about the maximum that can be injected in a single bunch. Figure 3 shows how the sidebands vary over a wide range of harmoniccavity power showing the transition between the shortbunch and the long-bunch regimes. With multibunch beams, the same instability is present but higher current may be injected. At these higher currents, the spectral bands widen to fill in the spectrum to give a fluctuating spectral density without obvious structure. With sufficiently high current, the beam dumps.

While the bunches are unstable, fluctuations of the rf in the harmonic cavity are rather strong, especially at high current. Although the impedance in that cavity is not higher than the main-cavity impedance, the fluctuations are more intense in the harmonic because of the fluctuation of beam loading arising from the fluctuation of the bunch's Fourier coefficient $\rho_{h}(t)$, and due to the particular configuration of servo loops for that cavity [7]. These loops are

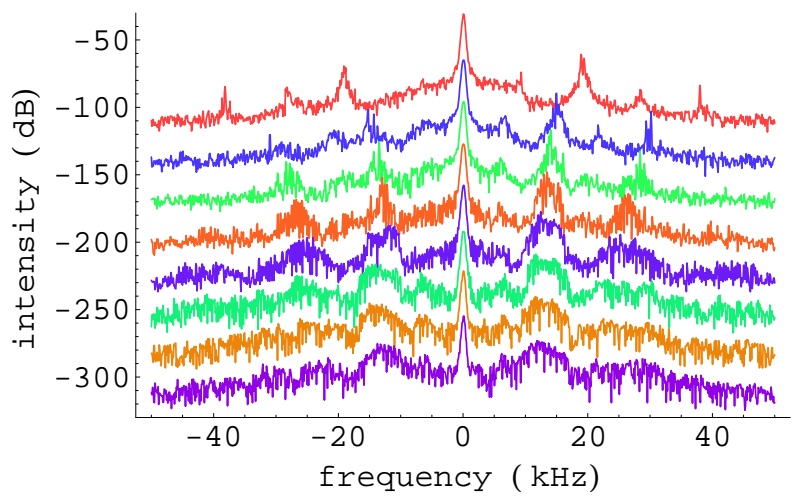

FIG. 3. (Color) Synchrotron sidebands of a single-bunch $205 \mathrm{~mA}$ beam about a high harmonic for several values of the harmonic-cavity voltage; the top trace is near zero, and the second to the bottom is near the optimum for stretching. The shortbunch trace at the top shows quadrupole-mode lines at $22 \mathrm{kHz}$ offset, and the long-bunch traces show broadened lines that fill the $10-15 \mathrm{kHz}$ range and its harmonics. not conventional phase- and level-regulation loops but are instead loops that level the rf power incident to the cavity and level the cavity field by controlling the phase of the amplifier power relative to the beam. In this arrangement, beam loading is essential to the operation of the loops. It is used because conventional loops cannot control the cavity adequately in the presence of stretched bunches [19].

There is also time structure exhibited by these spectra consisting of oscillations of the intensity of the sidebands with period approximately equal to the radiation damping time $(7 \mathrm{~ms})$. In Fig. 4 are data showing the intensity as a function of time of the synchrotron sideband centered at about $12 \mathrm{kHz}$ offset. The appearance of these spectral bands and their time structure seems to be due to the onset of relaxation of a quadrupolelike mode or a microwave instability and the fact that the threshold is smaller for stretched and overstretched bunches. So, while the average-current threshold for the microwave instability increases as the bunch is stretched due to its peak-current sensitivity, the average-current threshold for relaxation oscillation actually decreases as the bunch is stretched. The two thresholds are approximately equal for a stretched bunch.

The second feature of VUV beams that the code has helped us understand is the origin of two bumps in the stretched-bunch longitudinal beam response function on both sidebands of harmonics of the bunch repetition frequency (bunch harmonics) in a symmetric fill. The BRF $T_{m n}$ agrees closely with the beam transfer function $G_{m n}$ when measured about nonbunch harmonics of a multistretched-bunch beam (all traces of Fig. 5 except the top one), even at substantial currents. But a BRF measured about a bunch harmonic, such as the top trace of Fig. 5, has features distinctly and consistently different from the others - peaks typically at 6 and $12 \mathrm{kHz}$. Noise sidebands, which are sidebands modulated by the BRFs, also have these same peaks. The peaks are not particularly sensitive

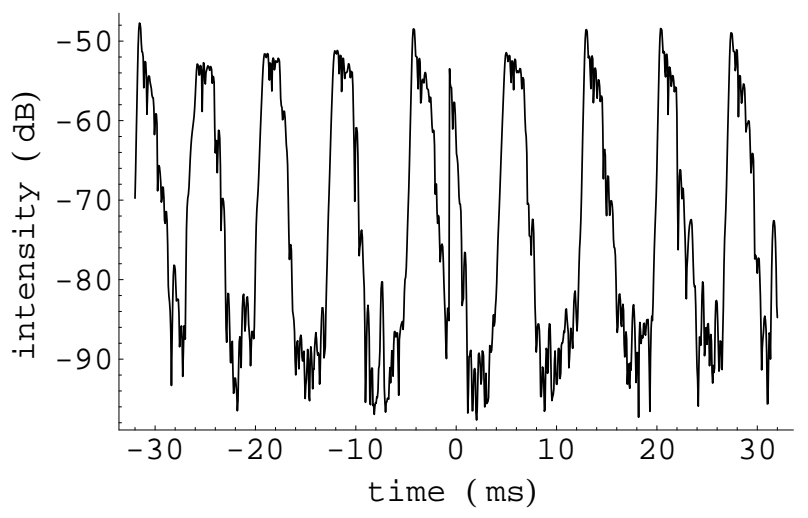

FIG. 4. The intensity as a function of time of the synchrotron sideband of the second to the lowest trace of Fig. 3 centered at about $12 \mathrm{kHz}$ offset taken with a spectrum analyzer at $3 \mathrm{kHz}$ resolution bandwidth. The discontinuity near the center of the horizontal axis is an artifact from the instrument. Machine parameters are given in Table I. 


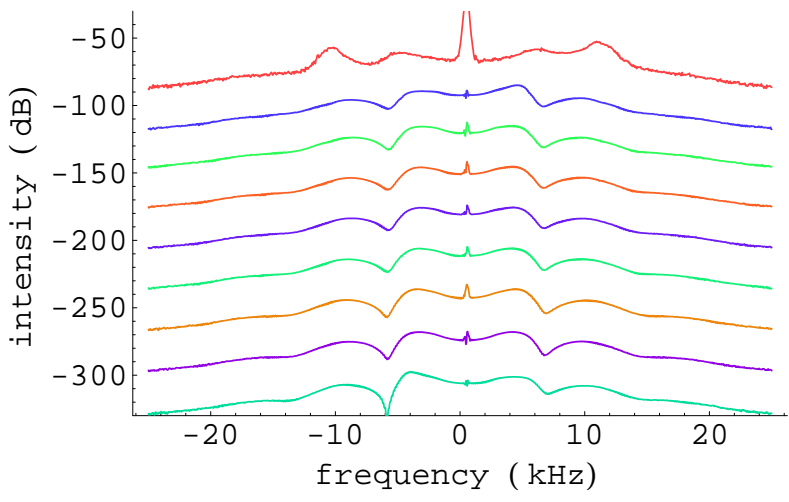

FIG. 5. (Color) Measured beam response functions of a $200 \mathrm{~mA}$ nine-bunch fully stretched beam about nine successive revolution harmonics in the NSLS VUV ring. The top trace is about the 54th revolution harmonic (sixth bunch harmonic) and lower traces are successive revolution lines. Machine parameters are given in Table I.

to gains and time constants in the servo loops of the rf system although the $6 \mathrm{kHz}$ peak changes with main-cavity tuning in a manner similar to the lower-frequency Robinson mode in the single-bunch single-cavity case [20].

These facts suggest that the HOM impedances have little impact on the nonbunch-harmonic BRFs of stretched bunches, which are the only impedances contributing to these response functions, and that the 6 and $12 \mathrm{kHz}$ features of bunch-harmonic BRFs have their origin in the high- $Q$ accelerating-mode impedances of the two cavities. But it remained for computer simulations to show that oscillations associated with the 6 and $12 \mathrm{kHz}$ features were dipolelike and quadrupolelike modes, respectively, and that each has considerable mode coupling. Furthermore, the code reproduced the short-bunch to long-bunch transition of the quadrupole mode instability, relaxation oscillations in the long-bunch regime, and, with mixed success, the prediction of spectra of unstable stretched bunches.

\section{SIMULATION OF STRETCHED BUNCHES}

Unstretched single-bunch beams in the VUV ring often show evidence of quadrupole instability, of which Fig. 3 is an example. As the bunch is stretched, the quadrupole mode decreases in frequency, as expected, and later merges with or becomes the broadened peaks tentatively associated with relaxation oscillations of a quadrupole mode or microwave instability excited by the broadband impedance. This was discussed in Sec. IV. It is of interest to see in detail in simulations the character of the instability associated with the broadened peaks and distinguish high- $Q$ driven effects from broadband impedance driven effects and how the two effects combine. This section discusses simulations of stretched bunches with and without high- $Q$ and broadband impedances and calculations of beam response functions. The results together form a rough picture of how impedances give rise to different features observed in the NSLS VUV ring (Sec. IV).
Simulations of this section assume a radiation damping time of $3.5 \mathrm{~ms}$, half the actual value listed in Table I. This reduced value is used to reduce the disparity in the time scales of the problem.

The rf potential for the optimum flat-bottomed potential well adjusts the main-cavity and higher-harmonic-cavity voltages and phases for zero net energy loss and zero first and second derivatives at $\phi=0$ [21]. In the VUV ring, these conditions have the main-cavity voltage and phase at $V_{9}=80 \mathrm{kV}$ and $\psi_{9}=74.2^{\circ}$ and the harmonic-cavity voltage and phase at $19.3 \mathrm{kV}$ and $-94^{\circ}$. The harmoniccavity servo loops are configured to maintain the cavity phase at $\psi_{36}=-90^{\circ}$, and the potential used in the simulations was chosen to be consistent with this fact. The harmonic-cavity voltage was chosen to be $V_{36}=19.7 \mathrm{kV}$. Some asymmetry in the computed Haïssinski distributions can be seen using these numbers. These and other machine parameters are listed in Table I. Detuning of the rf-cavity accelerating modes for beam-loading compensation was set to be proportional to the average current approximating what is actually used in machine operation. In the case of the main cavity, the proportionality is $50 \mathrm{kHz} / \mathrm{A}$; in the case of the harmonic cavity, it is $250 \mathrm{kHz} / \mathrm{A}$.

\section{A. Microwave instability}

The bunched-beam microwave instability $[22,23]$ is believed to be present in the VUV ring in short bunches at fairly low current $(10-30 \mathrm{~mA})$ in unstretched bunches and causes bunch lengthening at these currents and above. At a few hundred milliamps of single-bunch current, the bunch is 2 to 3 times larger than the small-current bunch length. The VUV ring can hold sufficient current that stretched bunches are microwave unstable as gauged by the Boussard criterion [22] and an estimate of the broadband impedance. A calculation of the threshold for the microwave instability in a stretched bunch by simulation gave a value given by the Boussard criterion [[15], Eq. (5.30)] to a precision of $15 \%$.

Simulations of microwave instability were done using VUV parameters (Table I). The broadband impedance is that of a $Q=1.118$ resonator with $\left|Z_{n} / n\right|=1.84 \Omega$ and with a resonant frequency $(2.0 \mathrm{GHz})$. Figure 6 is a plot of the distribution in phase space of an unstable bunch. Phase is left to right (leading edge of the bunch is to the right) while energy is from front to back.

Figure 7 shows simulated synchrotron sidebands at the 135th revolution harmonic for bunches at 300,350, and $400 \mathrm{~mA}$ beam current. At the highest current (top trace) there is a relatively uniform and intense spectral density indicative of rather disordered motion. The correlation with the features of Fig. 2 is not perfect, but the intense quasiuniform spectral density seen in the simulation is also present in spectra taken from other intense and/or overstretched beams in the VUV ring and is considered success of the simulation. 


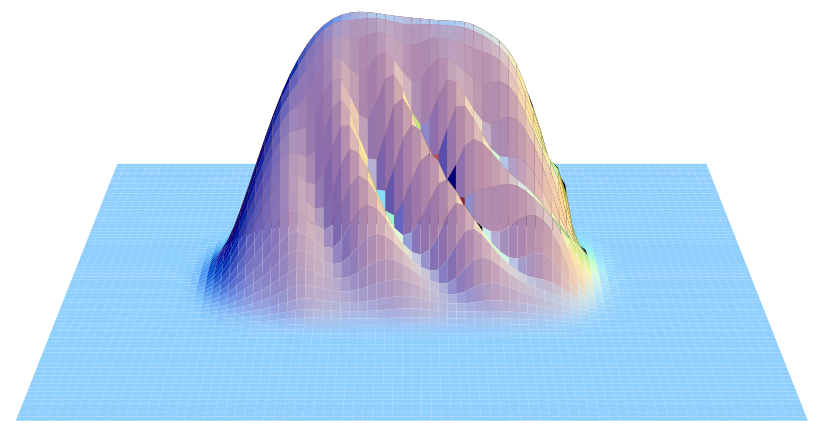

FIG. 6. (Color) Plot of a simulated $350 \mathrm{~mA}$ microwave-unstable fully stretched bunch in the NSLS VUV ring. The impedance is that of a $Q=1.118$ resonator with $\left|Z_{n} / n\right|=1.84 \Omega$ and resonant frequency 2.0 GHz. Phase increases from left to right with the leading edge of the bunch to the right. Energy increases from front to back. Machine parameters are given in Table I.

The bunch at $300 \mathrm{~mA}$ in the lower-current trace of Fig. 7 is microwave unstable but without bursting mode. It has sharply peaked synchrotron sidebands at a high offset $(32 \mathrm{kHz})$; the oscillation is nearly periodic (spectral density near these peaks is due to the transient associated with the start of the simulation). The line density shows a fast wave [24] with the $32 \mathrm{kHz}$ frequency moving across the bunch. When looking at the distribution in phase space, one sees that waves of modulation move across the lowenergy side of the bunch (e.g., Fig. 6) with the flow of the rf Hamiltonian towards the leading edge. Waves on this side are antidamped, i.e., they grow as they move with the flow. In contrast, the modulation on the high-energy side of the bunch damps. This imbalance in the damping rates permits the fast wave to dominate the modulation of the line density.

The frequency offset $\omega$ of the sideband is understood simply as $2 \pi$ times the velocity of this wave divided by the wavelength of the wave. The velocity of the wave is estimated as the velocity $d \phi / d t=p$ of particles in

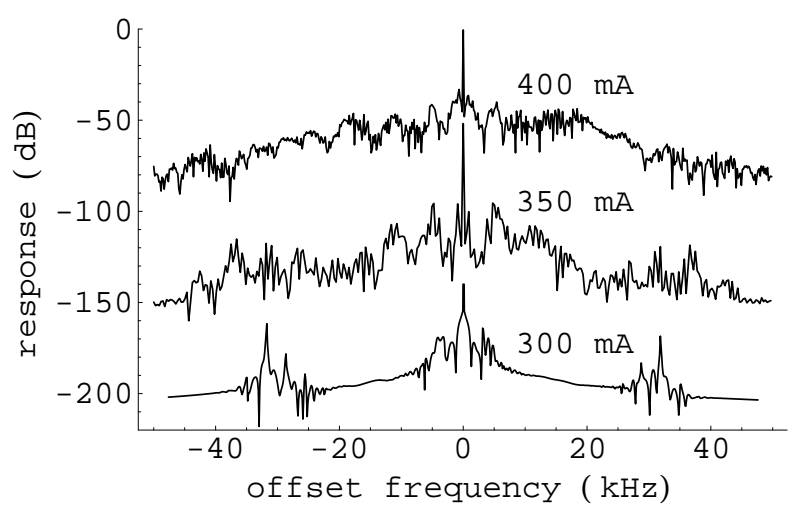

FIG. 7. Spectrum of simulated microwave-unstable bunches in the NSLS VUV ring at three beam currents. The ring impedance is that of a $Q=1.118$ resonator with $\left|Z_{n} / n\right|=1.84 \Omega$ and resonant frequency $2.0 \mathrm{GHz}$. Successive traces are displaced vertically $50 \mathrm{~dB}$. Machine parameters are given in Table I.

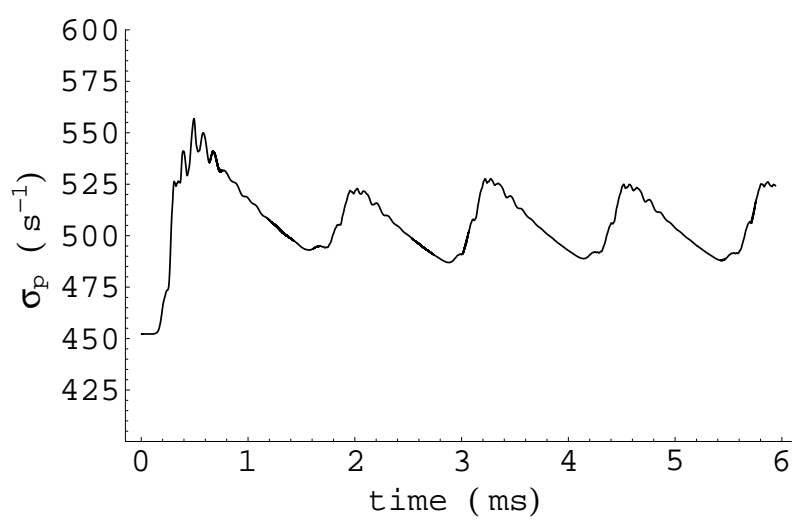

FIG. 8. Plot of the energy spread against time for a simulated microwave-unstable bunch in the NSLS VUV ring corresponding to the $400 \mathrm{~mA}$ trace of Fig. 7. The impedance is of a $Q=1.118$ resonator with $\left|Z_{n} / n\right|=1.84 \Omega$ and resonant frequency $2.0 \mathrm{GHz}$. Machine parameters are given in Table I.

phase space, where $p$ is evaluated at the edge of the bunch, i.e., $H_{\mathrm{rf}}(0, p)=p^{2} / 2=\sigma^{2}$, where $\sigma=\alpha \omega_{0} \sigma_{\epsilon}$ and $\sigma_{\epsilon}$ is the intrinsic fractional energy spread of the ring. The wavelength of the wave is the wavelength of the broadband impedance $2 \pi c / \bar{\omega}$ divided by the average radius of the ring $R=c / \omega_{0}$, where $\bar{\omega}$ is from Eq. (2.30) and $c$ is the speed of light. So the frequency of the sideband is

$$
\omega=\sqrt{2} \alpha \sigma_{\epsilon} \bar{\omega} .
$$

Using VUV numbers from Table I and $\bar{\omega}=2 \pi \times$ $1.8 \mathrm{GHz}$, this comes to $\omega=2 \pi \times 31 \mathrm{kHz}$, which is very close to the frequency seen in the simulation of Fig. 7. These lines have not been seen in spectra from the VUV ring, however. The microwave instability that is responsible for bunch lengthening in the VUV ring has frequency that is unlikely to be as low as was used in the calculations contained in this paper. It is more likely that the actual frequency $\omega$ is higher and observable only near higher revolution harmonics due to the low current at which the nonbursting mode occurs in the VUV ring.

At higher current, relaxation oscillations are present in simulations (Fig. 8). The intensity of the microbunching is greatest at the start of the simulation where the energy spread is artificially low (the natural machine energy spread). In later cycles, the instability revives before the energy spread fully damps to the natural energy spread resulting in less intense bursts.

\section{B. Simulations of longitudinal beam response functions}

About nonbunch harmonics, the longitudinal beam transfer function $G_{m n}[14,15]$, i.e., the beam response to an applied voltage in the absence of impedances, has the characteristic lobed structure whose lobes correspond to the terms of the series decomposition of $G_{m n}$ into a sum over the synchrotron harmonics [25]. In Fig. 9 is a measurement, which is of the beam transfer function 


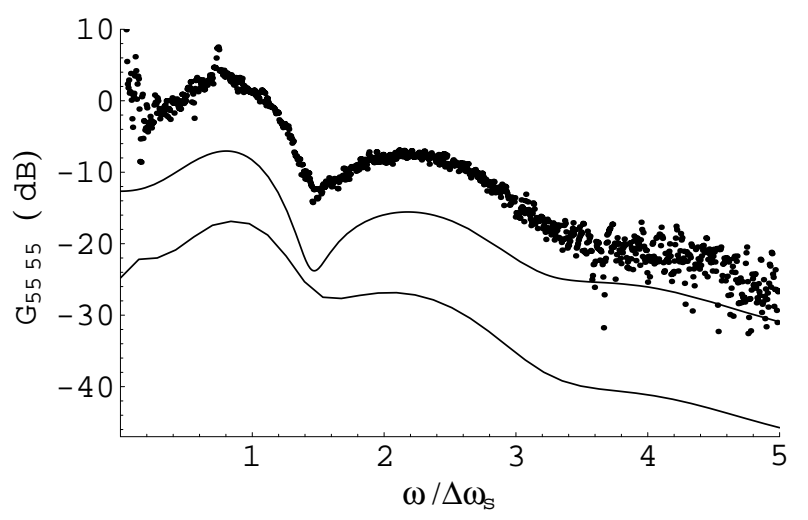

FIG. 9. Beam transfer function $G_{55} 55$ of a fully stretched single-bunch beam in the VUV ring. The data points are the beam response function $T_{55} 55$ measured at $13 \mathrm{~mA}$ approximating $G_{55} 55$; the middle trace is calculated using a method derived from Krinsky and Wang's [15] expression for their beam transfer function $F_{m n}=-G_{m n} / \kappa$ (see the text); the lower trace is calculated using the method described in Sec. III. The vertical scale has an arbitrary scale factor and the horizontal scale of the first and third traces $\left(\Delta \omega_{s}\right)$ was adjusted $20 \%$ to fit the second trace. Machine parameters are given in Table I.

of a fully stretched VUV beam at low current, and two calculations of the beam transfer function $G_{55} 55$ showing this structure. The first calculated response uses a method derived from Krinsky and Wang's [15] expression for the beam transfer function $F_{m n}=-G_{m n} / \kappa$. In this method [26], $F_{m n}$ is the Fourier transform

$$
F_{m n}(\Omega)=\frac{1}{i n} \int_{0}^{I} d \tau e^{i \Omega \tau} h_{m n}(\tau)
$$

where

$$
h_{m n}(\tau)=-\kappa \int_{0}^{\infty} d J\left\{g_{m n}, \Psi_{\mathrm{Ha}}\right\},
$$

$(J, \theta)$ is the action-angle canonical-coordinate pair with the constraint $\theta=-\omega_{s}(J) \tau$ in Eq. (5.3), $\omega_{s}=\omega_{s}(J)$ is the synchrotron frequency, $T_{s}=2 \pi / \omega_{s}, \Psi_{\mathrm{Ha}}$ is the Haïssinski distribution, $\kappa=e \alpha \omega_{0}^{3} / 4 \pi^{2} E_{0}$,

$$
\begin{aligned}
g_{m n}(J, \theta) & =\int_{0}^{2 \pi} e^{i\left[m \phi\left(J, \theta^{\prime}\right)-n \phi\left(J, \theta+\theta^{\prime}\right)\right]} d \theta^{\prime} \\
& \simeq 2 \pi J_{0}\left(r R_{m n}(\theta)\right),
\end{aligned}
$$

$\phi=\phi(J, \theta)$ is the $\phi$ coordinate as a function of the action-angle variables, $J_{0}$ is the Bessel function of the first kind, $R_{m n}(\theta)=\sqrt{m^{2}+n^{2}-2 m n \cos \theta}$, and, as above, the $\tau$ dependence of $R_{m n}(\theta)$ is in $\theta=-\omega_{s}(J) \tau$. But the derivations of Eq. (5.2) and $\mathrm{K} \& \mathrm{~W}$ 's expression from which Eq. (5.2) is derived neglect the potential-well distortion term and the FP terms as well as the nonlinear term of the VFP equation (2.11). For this reason, in this context, Eq. (5.2) is expected to be accurate only at lower currents, which is where Fig. 9 applies. It does, however, include all orders of synchrotron harmonics. The second calculated response of Fig. 9 uses the method described in Sec. III, i.e., the impulse-response simulation. These three response functions show good agreement.

The beam's response about harmonics of the bunch repetition frequency (top trace of Fig. 5), a response function that is distinctly different from the beam transfer function of Fig. 9 and the response near revolution harmonics that are not harmonics of the bunch repetition frequency (all traces of Fig. 5 except the top), suggests that the maincavity and harmonic-cavity fundamental rf modes are responsible for these peaks. These ideas were confirmed in a response-function calculation where the ring impedance includes the main-cavity and harmonic-cavity rf-mode impedances. Figures 10-12 are plots of simulated beam responses for 300,500 , and $800 \mathrm{~mA}$ beams with traces at different revolution harmonics. The results show the peaks clearly at 5 and $12 \mathrm{kHz}$, approximately where they exist in the top trace of the measured response Fig. 5 [compare with the fourth $\left(T_{55} 55\right)$ traces of Figs. 10-12].

The nature of the coherent motion associated with the 5 and $12 \mathrm{kHz}$ peaks was established by computing the time dependence of the first- and second-order moments of the bunch and comparing their intensities. The results are that there is much greater oscillation of the phase spread and energy spread at the $12 \mathrm{kHz}$ frequency than phase and energy, respectively, implying that the $12 \mathrm{kHz}$ peak is largely a quadrupole-mode oscillation. Oscillation of both dipole and quadrupole modes is present, however, so there is considerable mode coupling. The $5 \mathrm{kHz}$ feature is largely a dipole (center-of-mass) oscillation although, again, there is mode coupling. This is consistent with the calculations of Wang [10] and Bosch, Kleman, and Bisognano [11] showing that dipole-quadrupole mode coupling is significant in stretched bunches. So, the short-bunch quadrupole mode seems to retain its identity through the stretched-bunch regime as viewed through the beam response-function calculation-consistent with Fig. 3.

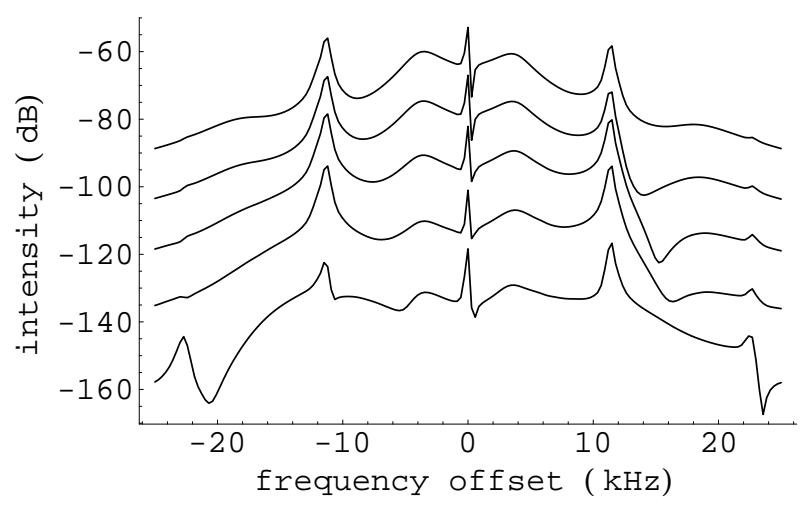

FIG. 10. Simulated beam response functions $T_{955}, T_{1855}$, $T_{3655}, T_{5555}$, and $T_{13555}$ (top to bottom) for a fully stretched $300 \mathrm{~mA}$ bunch in the VUV ring. Realistically detuned maincavity and harmonic-cavity impedances are included in the simulation. The vertical scale has an arbitrary scale factor. Machine parameters are given in Table I. 


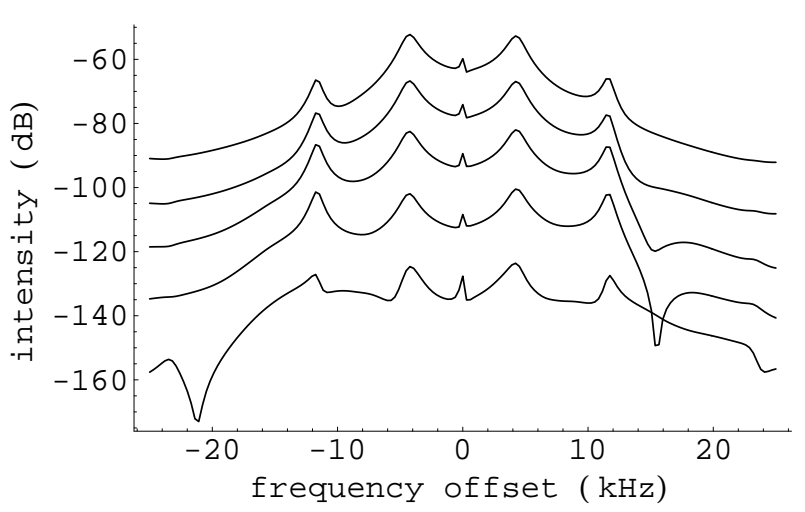

FIG. 11. Simulated beam response functions $T_{955}, T_{1855}$, $T_{3655}, T_{5555}$, and $T_{135} 55$ (top to bottom) for a fully stretched $500 \mathrm{~mA}$ bunch in the VUV ring. Realistically detuned maincavity and harmonic-cavity impedances are included in the simulation. The vertical scale has an arbitrary scale factor. Machine parameters are given in Table I.

There is a trend in the damping rates of the two modes evident in the three figures. As the current increases, the quadrupolelike mode becomes better damped while the dipolelike mode becomes more poorly damped. Simulations also predict that the dipolelike mode becomes unstable (Sec. VC) at about $200 \mathrm{~mA}$. In contrast, Fig. 5, which was taken from the VUV ring at $200 \mathrm{~mA}$, shows that the dipole mode is rather well damped. Furthermore, rigid-bunch calculations predict that the beam has an equilibrium-phase (reactive-Robinson-like) instability between 50 and $150 \mathrm{~mA}$. That this instability is not present in normal operation is perhaps due to the servo loop used in the harmonic-cavity rf system $[7,19]$ that is effectively beam feedback [6]. That loop may have sufficient bandwidth to provide adequate damping of the mode.

Although the agreement with measurements is not exact, these results resolved the long-standing question regarding the origin of the 5 and $12 \mathrm{kHz}$ peaks in beam response functions and noise sidebands, i.e., that the fundamental

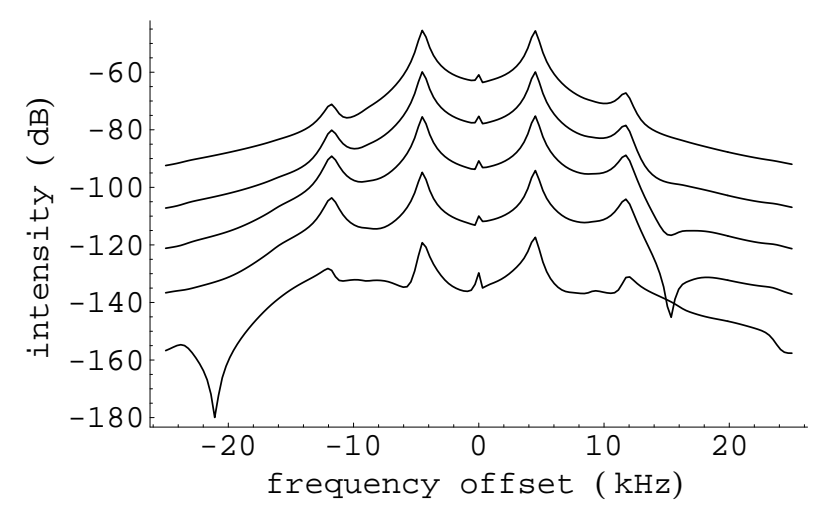

FIG. 12. Simulated beam response functions $T_{955}, T_{1855}$, $T_{3655}, T_{5555}$, and $T_{135} 55$ (top to bottom) for a fully stretched $800 \mathrm{~mA}$ bunch in the VUV ring. Realistically detuned maincavity and harmonic-cavity impedances are included in the simulation. The vertical scale has an arbitrary scale factor. Machine parameters are given in Table I.

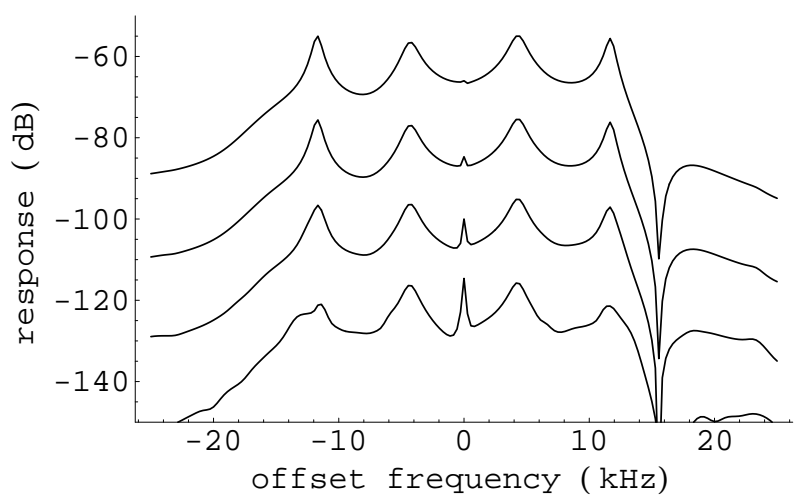

FIG. 13. Simulations of the response function $T_{55} 55$ of a $500 \mathrm{~mA}$ fully stretched bunch in a ring with VUV fundamental rf modes in the two cavities and no broadband impedance. The traces differ in their initial impulse $p_{0}$ in the momentum variable $p=d \phi / d t$ applied with the cosine- $\phi$ and sine- $\phi$ dependence as part of the impulse-based calculation of response functions (Sec. III). These impulses are, from top to bottom, $p_{0}=10,30,100$, and $300 \mathrm{~s}^{-1}$. Machine parameters are given in Table I.

rf modes of the two cavities are responsible for the two peaks, that the first is due to a dipolelike mode, the second is due to a quadrupolelike mode, gave some information about the degree of mode coupling involved, and that the broadened spectral bands of Fig. 2 are due to quadrupole instability with relaxation.

A last subject is briefly discussed in this section. When a bunch is externally excited by a continuous or transient force, resonances in the bunch's response may exhibit saturation even at low intensity of excitation. Saturation necessarily results in excitation-intensity-dependent beam response functions. Such effects are occasionally seen in the VUV ring. The method of Sec. III permits the calculation of beam response functions with varying intensity of the driving term. The initial kicks in energy are applied to the Haiissinski function so as to shift this function in the momentum variable $p=d \phi / d t$ by the $\phi$-dependent amounts $p_{0} \cos n \phi$ and $p_{0} \sin n \phi$ for the cosine and sine calculations, respectively (Sec. III). Figure 13 shows the $p_{0}$ dependence of simulated response functions of a $500 \mathrm{~mA}$ beam in the VUV ring with fundamental rf modes and no broadband impedance. Although the code does not simulate the continuous excitation of the bunch used in frequency-domain-based measurements, it is useful for estimating the onset of intensity-dependent effects.

\section{Instabilities driven by high- $Q$ modes}

In the next two simulations, only the fundamental rf modes of the main and harmonic cavities are present and the tunes of the main and harmonic cavities are realistically set for beam-loading compensation. At currents between about 50 and $200 \mathrm{~mA}$, the simulations predict instability. These thresholds are sensitive to the exact tuning of the two cavities. 


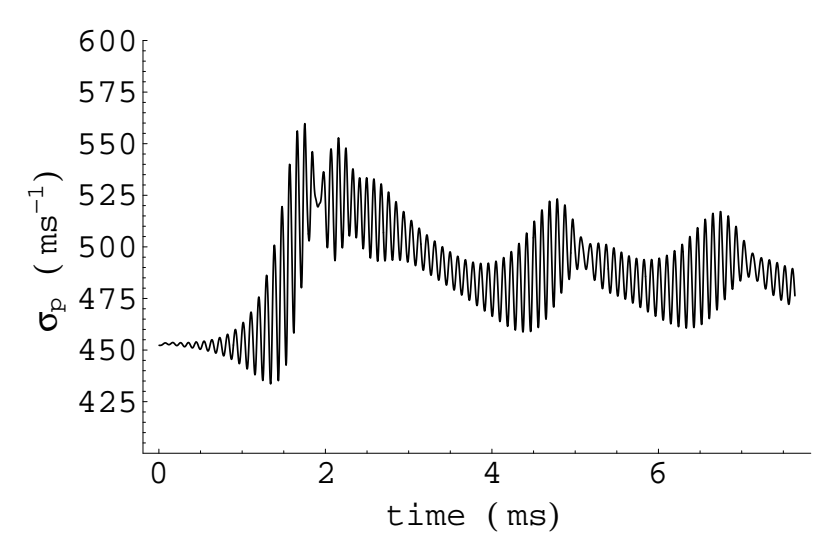

FIG. 14. Energy spread of a $200 \mathrm{~mA}$ fully stretched unstable bunch as a function of time. The ring impedance consists of main-cavity and harmonic-cavity fundamental rf modes realistically detuned for beam-loading compensation. Machine parameters are given in Table I.

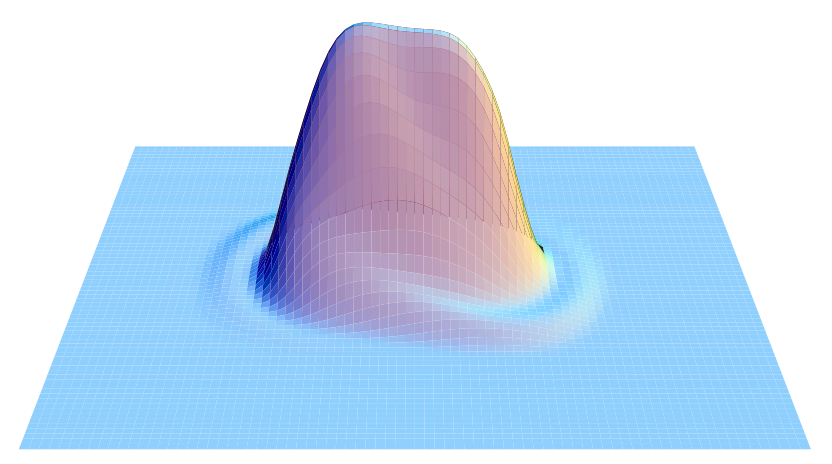

FIG. 15. (Color) Phase-space distribution of a $200 \mathrm{~mA}$ fully stretched unstable bunch that does not have a relaxation cycle. The ring impedance consists of main-cavity and harmoniccavity fundamental rf modes realistically detuned for beamloading compensation. Machine parameters are given in Table I.

At $200 \mathrm{~mA}$, the beam shows a weak quadrupolelike mode and relaxation oscillations. In Fig. 14 is plotted the energy spread of this bunch as a function of time showing the relaxation oscillations over several milliseconds; in Fig. 15 is presented a three-dimensional plot of the bunch at a point of the cycle at which the oscillation appears most intense; and in Fig. 16 is the spectrum of the bunch.

At $150 \mathrm{~mA}$, the intensity of the instability of the simulated bunch is much greater. Figure 17 shows a plot of the phase-space distribution after the oscillation becomes periodic (no relaxation) showing a striking twofold symmetry in phase space. It is curious that relaxation oscillations are absent at this current even though the instability is much more intense.

To summarize, Figs. $10-12$ and 17 illustrate that stretched bunches in the VUV ring show tendency to oscillate in a quadrupolelike mode at 10 or $12 \mathrm{kHz}$ - consistent with observations of the ring — and that this mode is driven by the high- $Q$ impedances of the rf cavities. But the spectra of this instability, while having $10-15 \mathrm{kHz}$

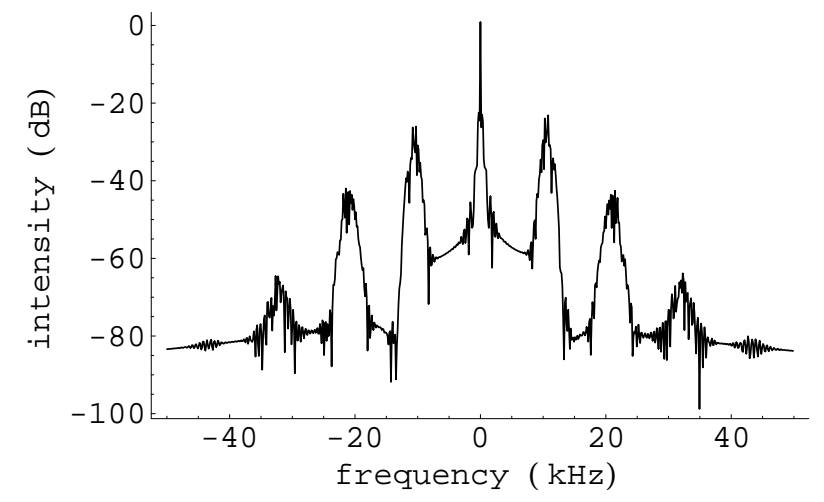

FIG. 16. Simulated spectrum about the 135th revolution harmonic of a $200 \mathrm{~mA}$ fully stretched weakly unstable bunch. The ring impedance consists of main-cavity and harmonic-cavity fundamental rf modes. Their tunes are realistically adjusted for beam-loading compensation. Machine parameters are given in Table I.

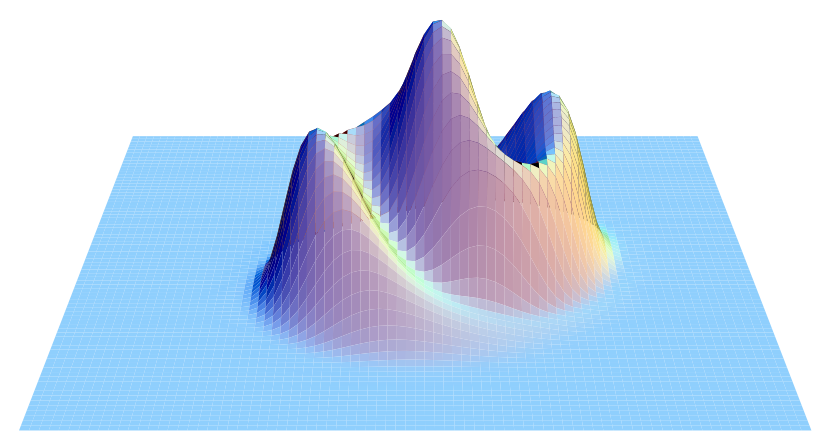

FIG. 17. (Color) Simulated phase-space distribution of a $150 \mathrm{~mA}$ fully stretched unstable bunch after the oscillations have steadied. The ring impedance consists of main-cavity and harmonic-cavity fundamental rf modes. Their tunes are realistically adjusted for beam-loading compensation. There is no relaxation cycle. Machine parameters are given in Table I.

sidebands, is still not clearly the origin of the instabilities of Figs. 2 and 3. We next look at simulations of spectra of combined broadband and high- $Q$ impedances.

\section{Combination of broadband impedance and high- $Q$ modes}

We know from the previous two sections that high- $Q$ modes drive instabilities in stretched bunches having spectra with multiple synchrotron harmonics that are typically broadened when there is relaxation. Also, broadband impedances drive instabilities that, when intense, have spectra with diffuse spectral density, when there is relaxation. In the absence of relaxation, both have spectra with sharp lines. The fundamental $\mathrm{rf}$ modes of the main and harmonic cavities can drive a quadrupolelike instability whose frequency, when stable or unstable, is in the same $10-15 \mathrm{kHz}$ range of the observed spectral bands of unstable stretched bunches in the VUV ring while also having diffuse spectral density. But the spectral bands with the diffuse 
background seen in Fig. 2 is not well reproduced in either of these types of simulations. We now look at simulations of instabilities at higher current in which there is a combination of microbunching driven by a broadband impedance (Sec. VA) and an unstable quadrupolelike mode driven by high- $Q$ impedances (Sec. V B).

In Fig. 18 are the energy spreads of three bunches as a function of time, one evolving in the high- $Q$ impedance alone, a second evolving in the broadband impedance, and a third evolving in both. The first case is stable while the second is microwave unstable with bunch lengthening and without bursting-mode behavior. The third case driven by both broadband and high- $Q$ impedances is unstable with relaxation. So, together the impedances push the bunch into the bursting-mode regime with an appearance very similar to that seen in a short-bunch simulation of Ref. [4].

Simulations with different broadband impedances were done to see how this changes the bunch spectra. In Fig. 19 are the beam spectra at $300 \mathrm{~mA}$ beam current for four different values of $\left|Z_{n} / n\right|$. The top trace is the spectrum of the bursting-mode trace of Fig. $18\left(\left|Z_{n} / n\right|=1.84 \Omega\right)$. The others have progressively higher impedance. The lines that are distinct in the top trace get progressively less distinct and increase slightly in frequency offset with increasing impedance. Also, the top trace has the lines that have nonzero widths that increase visibly in proportion to the synchrotron harmonic as in the high- $Q$-only case of Fig. 16. Lines on the other traces are not sufficiently distinct to see this because of the increasing intensity of the diffuse background.

While none of the traces of Fig. 19 resemble Figs. 2 or 3 closely, Fig. 19 does show the distributed spectrum and

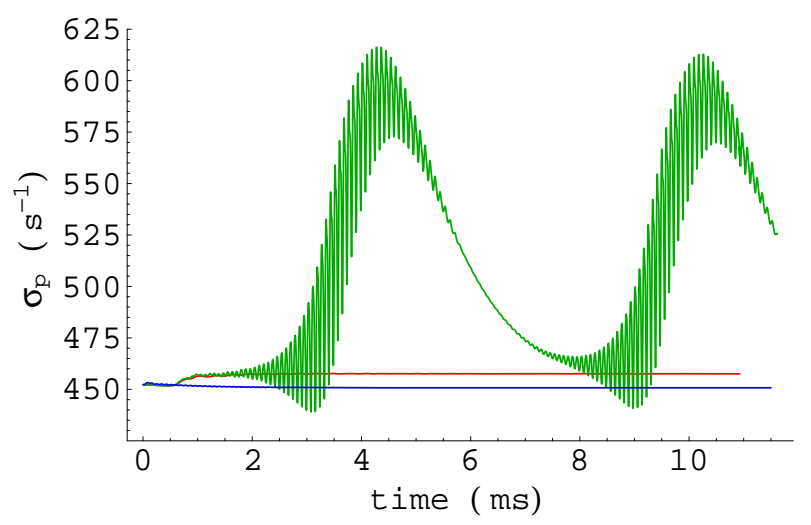

FIG. 18. (Color) Simulated energy spread of three $300 \mathrm{~mA}$ fully stretched bunches that differ in the ring impedances in which they propagate. From bottom to top the ring impedances are main-cavity and harmonic-cavity fundamental rf modes only (blue trace), broadband impedance consisting of a $Q=1.118$ resonator with $\left|Z_{n} / n\right|=1.84 \Omega$ and resonant frequency $2.0 \mathrm{GHz}$ only (red trace), and broadband impedance and high- $Q$ rf modes combined (green trace). The tunes of the rf modes are realistically adjusted for beam-loading compensation. Machine parameters are given in Table I.

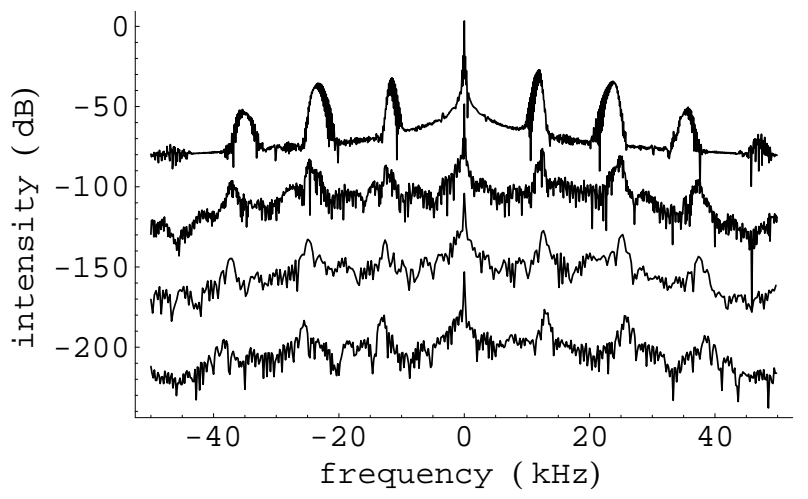

FIG. 19. Simulated spectra of $300 \mathrm{~mA}$ fully stretched unstable bunches propagating in a ring impedance consisting of maincavity and harmonic-cavity fundamental $\mathrm{rf}$ modes and broadband impedances consisting of a $Q=1.118$ resonator with varying $\left|Z_{n} / n\right|$ and resonant frequency $2.0 \mathrm{GHz}$. From top to bottom the traces have $\left|Z_{n} / n\right|$ of $1.84,2.32,2.48$, and $2.8 \Omega$. The frequency is offset from the 135th revolution harmonic. The tunes of the rf modes are realistically adjusted for beam-loading compensation. Successive traces are displaced $50 \mathrm{~dB}$. Machine parameters are given in Table I.

relaxation oscillations that are seen in intensely unstable VUV bunches.

\section{CONCLUSION}

Computational methods and a code were developed for the integration of the VFP equation, which follows closely, and extends, the work of W\&E [1], that tracks the particle distribution function in phase space under the influence of broadband and high- $Q$ impedances. A method for the calculation of beam response functions that includes the beam-induced voltages acting back on the bunch was also described and demonstrated in simulations.

The behavior of certain single-bunch instabilities present in stretched bunches in the VUV ring was then described. These instabilities often have distributed spectral density that has continuity between a quadrupole instability present in short bunches and a spectral band present in stretched bunches. Characteristics of measured BRFs of multibunch modes were described. The multibunch mode that does not interact with the rf system has a BRF that looks like the bare BTF, i.e., as though there was no ring impedance interacting with the bunches. In contrast, BRFs of those modes interacting with the rf system have distinctive features whose origins were more clearly understood with the help of simulations reported here.

Simulations of stretched bunches unstable in a ring impedance containing high- $Q$ or broadband impedances or both were presented. These simulations give some information on what observable consequences different types of impedances generate in a beam. In particular, the code was used to calculate saturation of microwave instability driven by a broadband impedance. Similar 
calculations for instabilities driven by high- $Q$ impedances of the fundamental rf modes of the main and harmonic cavities were performed. Then instabilities driven by both high- $Q$ and broadband impedances were simulated to look at how beam spectra varied with beam current and broadband impedance. These simulations established that unstable quadrupolelike modes are present in stretched bunches under the influence of the high- $Q$ impedances of the cavities, that relaxation is often present with these instabilities, and that there is considerable mode mixing. Calculations of BRFs also showed that a dipolelike mode and the quadrupolelike mode both contribute peaks in the BRFs ( 5 and $12 \mathrm{kHz}$ ), accounting for these previously unexplained features.

Although this study falls short of a quantitative understanding of the instabilities seen in the VUV ring and the impedances that drive them, significant progress was made. Calculations of spectra and response functions using more realistic broadband impedances are needed. Such investigations may lead to a more detailed and precise understanding of these long bunches and their instabilities. The physics of saturation of instabilities driven by combined high- $Q$ and broadband impedances is a subject worthy of further investigation.

\section{ACKNOWLEDGMENTS}

The author would like to thank Jiunn-Ming Wang, Sam Krinsky, and Bob Bosch for helpful discussions. This work was performed under the auspices of the U.S. Department of Energy under Contract No. DE-AC02-98CH10886.

[1] R. Warnock and J. Ellison, in Proceedings of the 2nd ICFA Advanced Accelerator Workshop on the Physics of High Brightness Beams, Los Angeles, CA, 1999 (World Scientific, Singapore, 2000).

[2] B. V. Podobedov, Ph.D. thesis, Stanford University, 1999.

[3] M. Migliorati, L. Palumbo, G. Dattoli, and L. Mezi, Nucl. Instrum. Methods Phys. Res., Sect. A 437, 134 (1999).

[4] R. Warnock, K. Bane, and J. Ellison, in Proceedings of the 7th European Particle Accelerator Conference (EPAC2000), Vienna, 2000, http://accelconf.web.cern.ch/ AccelConf/e00/index.html.

[5] C. Limborg and J. Sebek, Phys. Rev. E 60, 4823 (1999).
[6] Y. Miyahara, S. Asaoka, A. Mikuni, and K. Soda, Nucl. Instrum. Methods Phys. Res., Sect. A 260, 518 (1987).

[7] R. Biscardi, S. L. Kramer, and G. Ramirez, Nucl. Instrum. Methods Phys. Res., Sect. A 366, 26 (1995).

[8] T.-S.F. Wang, in Proceedings of the 15th International Conference on High Energy Accelerators, Hamburg, Germany, 1992, edited by J. Rossbach (World Scientific, River Edge, NJ, 1993).

[9] J. S. Berg and F. Ruggiero, in Proceedings of the 17th Particle Accelerator Conference, Vancouver, Canada, 1997, edited by M. Comyn, M. Craddock, M. Reiser, and J. Thomson (IEEE, Piscataway, NJ, 1998), Vol. 2, pp. 17121714 .

[10] T. S. F. Wang, Part. Accel. 34, 105 (1990).

[11] R. A. Bosch, K. J. Kleman, and J. J. Bisognano, Phys. Rev. ST Accel. Beams 4, 074401 (2001).

[12] O. Naumann and J. Jacob, in Proceedings of the 6th European Particle Accelerator Conference, Stockholm, Sweden, 1998, edited by S. Myers, L. Liijeby, C. Petit-Jean-Genaz, J. Poole, and K.-G. Rensfelt (IOP Publishing, Philadelphia, PA, 1998), pp. 787-789.

[13] J. Haissinski, Nuovo Cimento Soc. Ital. Fis. 18B, 72 (1973).

[14] E. N. Shaposhnikova, CERN Report No. SL-94-19-RF, 1994.

[15] S. Krinsky and J. M. Wang, Part. Accel. 17, 109 (1985).

[16] R. Talman (unpublished lecture notes).

[17] R. Talman, Superconducting Super Collider Laboratory Technical Report No. SSCL-Preprint-187, 1993.

[18] A. W. Chao, Physics of Collective Beam Instabilities in High-Energy Accelerators (Wiley, New York, 1993).

[19] N. Towne, in Proceedings of the 1999 Particle Accelerator Conference, New York, edited by A. Luccio and W. MacKay (IEEE, Piscataway, NJ, 1999), Vol. 2, pp. 10311033.

[20] N. Towne and J.-M. Wang, Phys. Rev. E 57, 3461 (1998).

[21] A. Hofmann and S. Myers, in Proceedings of the XI International Conference On High Energy Accelerators, Geneva, 1980, edited by W. Newman (Birkhauser-Verlag, Basel, Switzerland, 1980).

[22] D. Boussard, CERN Report No. MPS/DL-75/5, 1975.

[23] J.-M. Wang, Phys. Rev. E 58, 984 (1998).

[24] J.-M. Wang, in US/CERN Accelerator School, Capri, Italy, 1988, edited by M. Month and S. Turner, Lecture Notes in Physics No. 343 (Springer-Verlag, Berlin, 1989); BNL Report No. 42939.

[25] A. N. Lebedev, At. Energ. 25, 100 (1968) [Sov. J. At. Energy 25, 851-856 (1968)].

[26] N. Towne, in Proceedings of the 2001 Particle Accelerator Conference, Chicago, Illinois (to be published). 